\title{
Agreement Between Face-to-Face and Free Software Video Analysis for Assessing Hamstring Flexibility in Adolescents
}

\author{
José A. Moral-Muñoz, ${ }^{1}$ Bernabé Esteban-Moreno, ${ }^{2}$ Manuel Arroyo-Morales, ${ }^{3}$ \\ Manuel J. Cobo, ${ }^{4}$ and Enrique Herrera-Viedma ${ }^{5}$ \\ Departments of ${ }^{1}$ Library Science; and ${ }^{2}$ Physical Therapy, University of Granada, Granada, Spain; ${ }^{3}$ Department of Physical \\ Therapy, University of Granada, Granada, Spain; ${ }^{4}$ Department of Computer Science, University of Cádiz, Cádiz, Spain; and \\ ${ }^{5}$ Department of Computer Science and Artificial Intelligence, CITIC-UGR (Research Center on Information and \\ Communications Technology), University of Granada, Granada, Spain
}

\begin{abstract}
Moral-Muñoz, JA, Esteban-Moreno, B, Arroyo-Morales, M, Cobo, MJ, and Herrera-Viedma, E. Agreement between face-to-face and free software video analysis for assessing hamstring flexibility in adolescents. J Strength Cond Res 29 (9): 2661-2665, 2015-The objective of this study was to determine the level of agreement between face-to-face hamstring flexibility measurements and free software video analysis in adolescents. Reduced hamstring flexibility is common in adolescents ( $75 \%$ of boys and $35 \%$ of girls aged 10). The length of the hamstring muscle has an important role in both the effectiveness and the efficiency of basic human movements, and reduced hamstring flexibility is related to various musculoskeletal conditions. There are various approaches to measuring hamstring flexibility with high reliability; the most commonly used approaches in the scientific literature are the sit-and-reach test, hip joint angle (HJA), and active knee extension. The assessment of hamstring flexibility using video analysis could help with adolescent flexibility follow-up. Fifty-four adolescents from a local school participated in a descriptive study of repeated measures using a crossover design. Active knee extension and HJA were measured with an inclinometer and were simultaneously recorded with a video camera. Each video was downloaded to a computer and subsequently analyzed using Kinovea 0.8.15, a free software application for movement analysis. All outcome measures showed reliability estimates with $\alpha>0.90$. The lowest reliability was obtained for HJA ( $\alpha=0.91)$. The preliminary findings support the use of a free software tool for assessing hamstring flexibility,
\end{abstract}

Address correspondence to José A. Moral-Muñoz, jamoral@ugr.es.

29(9)/2661-2665

Journal of Strength and Conditioning Research

(C) 2015 National Strength and Conditioning Association offering health professionals a useful tool for adolescent flexibility follow-up.

KEY WoRDS face-to-face clinical assessment, free software video analysis, hamstrings flexibility, teenager

\section{INTRODUCTION}

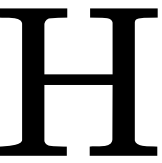

amstring length has an important role in both the effectiveness and the efficiency of basic human movements, such as walking and running (12). Reduced hamstring flexibility is common in adolescents; $75 \%$ of 10 -year-old boys and $35 \%$ of 10 -year-old girls have reduced hamstring flexibility (6). This poor hamstring flexibility is related to problems in executing and sustaining motor activities in daily life (4). Clinical observations have suggested that limited hamstring flexibility is associated with various musculoskeletal conditions, including specific disorders of the lumbar spine, such as low-back pain (32) and changes in the lumbopelvic rhythm (11). Likewise, in sports therapy, short hamstrings are related to muscle strains (27), a reduction in athletic performance (18) and the development of patellar tendinopathy and patellofemoral pain (19).

Various studies have been conducted to develop methods for measuring hamstring flexibility, and most of these methods are related to the sit-and-reach test (SRT) $(7,17)$, hip joint angle (HJA) (1), and active knee extension (AKE) (29). Because the SRT is relatively easy to administer, the validity and reliability of this test and modified versions of it have been tested $(7,22,24)$. However, the SRT is multifaceted in that the score can be affected by several spine angles, and it may not provide an accurate assessment of hamstring flexibility $(17,25)$. Consequently, SRT scores vary among individuals, and there are gender differences in the scores $(21,22,35)$.

The use of devices and software in medicine is increasing. The use of telecommunications and computer technologies to evaluate and deliver treatments for improving disability is termed telehealth (23). The development of this type of 
system has been attributed to various factors: the demand by health care users for novel forms of treatment (10), the globalization of health systems (14), and pressure to reduce health care costs, in addition to the major advances in information and communication technologies (31).

Various published studies have used measurement software to evaluate flexibility, movement or both $(5,8,9,16,24,25)$. Mier et al. $(24,25)$ used the proprietary software Dartfish ProSuite v4.0 software (Dartfish, Atlanta, GA, USA) in their studies. In these studies, the accuracy and feasibility of video analysis was evaluated using hamstring, spine, and pelvic flexibility measurements. In other studies, a custom-made MATLAB Software program (The MathWorks, Inc., Natick, Mass.) was used to estimate the movement of the low back in lifters (8) and workers in a field situation (9). Furthermore, nonspecific software has been used in several studies (e.g., Adobe Premier Pro, Adobe Photoshop) $(5,16)$.

The adaptation of software to evaluate the hamstring flexibility could be difficult in the case of the SRT because of the necessity of a new box model or a camera in the sagittal position to record the test. However, HJA and AKE also present some weaknesses. Hip joint angle involves whole-body motion; therefore, this measurement could be influenced by different factors; in contrast, there is no negative influence of pelvic position and stability in the scores obtained (1). AKE needs to be conducted under controlled conditions; the test depends on strict body stabilization and a well-defined and easily observed end point of motion (13).

Therefore, it is interesting to analyze the available tools for implementing new measurement approaches. In this case, the use of General Public License (GNU) software allowed for the development of a more applicable measurement method. GNU is a free copyleft license for software, meaning that the software is freely shared. The use of this software reduces the costs of implementing a video analysis system.

There are various GNU software available on the Internet, such as PhysMo (3) and LongoMatch (20), but the most used in the scientific literature and the easiest to use is Kinovea. Therefore, there are several recent articles in which Kinovea has been used, such as Balsalobre-Fernández et al. (2) where it was used to measure the flight time of vertical jumps, Telfer et al. (33) that used it to measure foot kinematics during gait, and Ogueta-Alday et al. (26) that used it to measure contact and flight times during treadmill running.

We hypothesized that the inclusion of free software video analysis as a tool in the arsenal of strength and conditioning professionals could be a reliable method for the assessment of hamstring flexibility in clinical practice. That tool could be useful for the coaches or professional practitioners to test the effectiveness of an exercise program for improving hamstring flexibility deficits. This study could demonstrate the highly reliability of free software video analysis in range motion assessment that could be applied to other joints.
The purpose of this study was to determine the level of agreement between face-to-face hamstring flexibility measurements and free software video analysis in adolescents.

\section{Methods}

\section{Experimental Approach to the Problem}

A descriptive crossover design was used to study the criterion validity and interrater reliability of the video analysis of hamstring flexibility. The objective of this study was to determine the level of agreement between face-to-face hamstring flexibility measurements and free-software video analysis in adolescents. Fifty-four adolescents from a local school were randomly allocated to be measured using both methods. Active knee extension and HJA measurements were used. Hip joint angle and AKE assessments are easily adaptable to on-line systems and do not require a health care practitioner to be conducted, as these assessment are more suitable and easy to administer and to record with a video analysis system in any place without special materials. These tests are described in the following sections.

\section{Subjects}

Before testing, the study procedures were discussed with the parents and subjects together. Parents, as legal guardian of participants, who agreed to the procedures read and signed an informed consent form written according to JSCR guidelines, previously approved by the university's institutional review board. Fifty-four adolescents (34 girls and 20 boys), who were students from a local school, participated in this study (age: $14.31 \pm 1.37$ years [ $12-18$ years], height: 162.91 $\pm 8.50 \mathrm{~cm}$, weight: $53.80 \pm 8.93 \mathrm{~kg}$, and body mass index: $20.19 \pm 2.33$ ). Before conducting the measurements, the Physical Activity Readiness Questionnaire for Everyone (PAR-Q+) form (34) was completed by the participants and parents. None of the participants had any musculoskeletal injuries or conditions that could alter performance, and none reported muscle soreness from physical activity on the test day.

\section{Procedures}

The subjects who wanted to take part changed into loose shorts and removed their shoes and were weighed using an InBody 770 body composition analyzer (InBody Co., Seoul, Korea); height was measured using a Leicester Height Measure Mk II (Child Growth Foundation, Chiswick, United Kingdom). The data collection was conducted in a classroom as part of the Physical Education class and without an audience; the presence of classmates could influence the subject's concentration in measurements. Hip joint angle and AKE measurements were performed by 2 examiners at random for controlling order effects because of the repeated-measures design. The data collection was carried out by 2 examiners to reduce study time. All subjects completed the 2 tests and were simultaneously recorded with a video camera. Once we completed the session, we tested the interrater and intrarater reliability of the video recordings. The data from the video recordings were 
compared with the examiner data. To test the intrarater reliability, the examiner reviewed the video 1 month after the initial examination and made a new assessment; 1 month is generally considered sufficient to limit test-retest bias (30).

\section{Active Knee Extension Test}

The AKE test (29) was used; the subject was supine with both the knee and hip positioned in $90^{\circ}$ flexion. A mark was made on the tibial crest, $10 \mathrm{~cm}$ distal to the tibial plateau for the placement of the digital inclinometer. Then, the participant was asked to actively extend the lower leg until the point of limitation. Hip flexion was maintained with the use of a support on the posterior thigh. The subject's AKE measurement was recorded 3 times using an AcumarTM (ACU 360) digital inclinometer (Lafayette Instrument Co., Lafayette, IN, USA) placed over a marked position on the subject's anterior leg, 10 $\mathrm{cm}$ distal to the tibial plateau. The examiner, located on the opposite side to the camera, read the inclinometer LCD and stated the measurement, whereas another researcher recorded the results as an average of the 3 measurements.

\section{Hip Joint Angle Test}

The HJA test (1) was used; using a standard sit-and-reach box (12-in $[30.5 \mathrm{~cm}]$ high) (Lafayette Instrument Co), we measured the angle of forward inclination of the sacrum and pelvis relative to the horizontal plane at the point of maximum forward bending. The examiner, placed behind the subject's back, recorded the measurement 3 times using an AcumarTM (ACU 360) digital inclinometer (Lafayette Instrument Co) placed on the sacrum at the level of the posterior superior iliac spine. The HJA is formed between the pelvis and the femur during the end point of maximum forward reach with the knees in voluntary full extension. The HJA score was calculated as the supplementary angle reported by the sacral horizontal angle.

\section{Video Analyses}

A professional high-speed digital video camera (Sony HXRNX5U NXCAM; Sony Corp., Minato, Tokyo, Japan) was set on a tripod and placed so that the lens was approximately 3 $\mathrm{m}$ from the edge of the examining table, according to a previously published procedure (24). The lens and table surface were adjusted to the same distance from the floor. A level was used to adjust the horizontal plane of the lens and table so that both were parallel to the floor before each test. Frame rate was selected $(1,200 \mathrm{~Hz})(26)$, and the tripod was placed in a location that allowed the hip and knee joints to be centered on the LCD; the lens focal length was adjusted so that the hip joint and the thigh and knee of the raised leg could be viewed. Each video was downloaded to a computer and subsequently analyzed using Kinovea 0.8 .15 (2). Kinovea is a free software application for the analysis, comparison, and evaluation of sports and training.

In the Kinovea free software application, a full leg and hip angle raise was visually determined frame by frame until the greatest distance achieved was identified. The frame was frozen, and the angle tool was used to determine AKE and HJA. The axis of rotation of the angle tool was aligned with the mark, previously painted on the skin, on the lateral epicondyle of the femur and greater trochanter, respectively. For AKE, 1 arm of the angle was aligned with the external malleolus and the other arm was aligned with the greater trochanter. For HJA, 1 arm of the angle was aligned parallel to the table surface and the other arm was aligned parallel to the sacrum.

\section{Statistical Analyses}

The agreement between face-to-face and video analysis assessments was analyzed using the Bland and Altman "limits of agreements" statistic for continuous variables and by applying Cronbach's $\alpha$ and the intraclass correlation coefficient (ICC) ( $\rho$ ). Two-way random-effects ICCs $(\rho)$ and their confidence intervals were calculated for the inter- and intrarater reliability trials. SPSS version 20.0 (IBM, Corp., Armonk, NY, USA) was used for the statistical analyses. In accordance with previous studies (15), $\rho$ less than 0.4 was considered poor reliability, 0.4 to 0.75 was considered fair to good reliability, and greater than 0.75 was considered excellent reliability.

\section{Results}

The data from the 54 participants were analyzed to obtain the reliability values for the experiment. The interrater reliability between tester $\mathrm{A}$ and $\mathrm{B}$ in the face-to-face approach was obtained. The AKE and HJA tests have very good reliability (AKE right: $\alpha=0.98$, AKE left: $\alpha=0.98$, and HJA: $\alpha=0.96)$. The data used in the following analysis may be from either tester.

Table 1 provides the $\alpha$ reliability estimate for each outcome measure between the face-to-face and video analysis. All of the measures showed excellent reliability $(\alpha>0.90)$. The lowest reliability was obtained for the HJA measurement $(\alpha=0.91)$. 
TABLE 2. Test-retest reliability for AKE and HJA.*

\begin{tabular}{lccc}
\hline Variable & ICC $(\rho) \dagger$ & $95 \%$ Cl of ICC & $\begin{array}{c}\text { Cronbach's } \alpha \text { reliability } \\
\text { estimate }\end{array}$ \\
\hline AKE right & 0.97 & 0.96 to 0.99 & 0.97 \\
AKE left & 0.98 & 0.97 to 0.99 & 0.98 \\
HJA & 0.98 & 0.96 to 0.99 & 0.98
\end{tabular}

${ }^{\star} \mathrm{ICC}=$ intraclass correlation coefficient; $\mathrm{Cl}=$ confidence interval; $\mathrm{AKE}=$ active knee extension; HJA = hip joint angle.

†ICC $(\rho)$ was calculated using a 2-way mixed-effects model.

the follow-up of adolescent patients. Health services can use the system to prevent future alterations in young people. This type of health service seems to be increasing (23), and there are several software solutions available that can affect the system costs. This study makes an initial contribution to the development of systems for reducing the cost of preventive health care.

The inclusion of an assessment in real-time on the Inter-

After an interval of 1 month, high test-retest reliability was demonstrated for the AKE and HJA tests using video analysis, which is shown in Table 2. The $\alpha$ reliability estimate is excellent in all measures with $\alpha>97$.

\section{Discussion}

The results of this study demonstrate that video analysis can be used as an acceptable tool for measuring hamstring flexibility. The values support the reliability of both tests using this method of measuring. The validity of the assessment was established by comparing the results obtained from the video analysis with those obtained from the face-to-face method. The originality of the study lies in the use of Kinovea, GNU software available to any user that can be used to analyze a video or image without high costs.

The high concordance between the face-to-face and video analysis systems for measuring hamstring flexibility was similar to that obtained in previous studies for the video analysis of hamstring flexibility and the use of a computer software angle tool for measuring spine and pelvic flexibility $(24,25)$.

This tool could be useful for measuring and following up reduced hamstring flexibility in adolescents. The efficiency of basic human movements, such as walking and running, could be affected if adolescents maintain shorter hamstrings. As indicated above, limited hamstring flexibility is associated with various musculoskeletal alterations, including specific disorders of the lumbar spine, such as low-back pain (32) and changes in the lumbopelvic rhythm (11). To prevent these alterations, it is important to use measurement tools that support an adequate stretching program.

Constant technological improvements and the increasing need to reduce health care costs have focused research attention on the potential of telehealth (28). Telehealth systems may be a useful option for people who do not have easy access to health services (30). Furthermore, telehealth could be a powerful tool in preventive health; it could be used to facilitate contact between health professionals and different population segments.

In this study, the system could allow health services and schoolteachers to be in contact. This approach helps with net could be an interesting topic of future study. The implementation of these types of assessment tools could be useful. Nevertheless, it is necessary to develop encrypted software to prevent security breaches. Currently, there is no clear law on the treatment of this type of information over the Internet. Health service users will need to have the technological means to carry out these measures. The implementation of these types of systems could be a limitation because of the current economic climate.

Our study was limited to adolescents registered in a local school. It is difficult to access different groups of adolescents from various areas, and it is sometimes impossible to coordinate a study with the teaching plan. Another limitation of this study could be the need to perform the measurements in a real situation. This study was conducted in a controlled environment with people who understood the design. Replication studies in different situations with various types of equipment and untrained users are needed to validate these findings.

\section{Practical Applications}

The preliminary findings support the use of a free software video analysis tool to evaluate hamstring flexibility in a clinical or adolescent population, preventing future risk of back and hamstring injuries. Our study demonstrates that Kinovea software is a highly reliable and objective method for evaluation of hamstring flexibility. That tool could be useful for the coaches or professional practitioners to test the effectiveness of an exercise program for improving hamstring flexibility deficits. To get accurate and reliable measurements, we have to be consistent in procedure. The inclusion of an assessment in realtime on the Internet could be useful for strength and conditioning professional to facilitate the follow-up of hamstring flexibility in a training session. Furthermore, this method could be considered as an accurate alternative to the handheld inclinometer or goniometer for assessing range of motion of other joints.

\section{ACKNowledgments}

J.A. Moral-Muñoz holds an FPU scholarship (AP2012-1789) from the Spanish Ministry of Education. 


\section{REFERENCES}

1. Ayala, F, Sainz de Baranda, P, Ste-Croix, C, and Santonja, F. Reproducibility and concurrent validity of hip joint angle test for estimating hamstring flexibility in recreationally active young men. $J$ Strength Cond Res 26: 2372-2382, 2012.

2. Balsalobre-Fernández, C, Tejero-Gonzalez, C, Campo-Vecino, J, and Bavaresco, N. The concurrent validity and reliability of a lowcost, high-speed camera-based method for measuring the flight time of vertical jumps. J Strength Cond Res 28: 528-533, 2013.

3. Barraclough, J. PhysMo-Video motion analysis package. SourceForge, 2011.

4. Bergstrom, G, Aniansson, A, Bjelle, A, Grimby, G, LundgrenLindquist, B, and Svanborg, A. Functional consequences of joint impairment at age 79. Scand J Rehabil Med 17: 183-190, 1985.

5. Boden, BP, Torg, JS, Knowles, SB, and Hewett, TE. Video analysis of anterior cruciate ligament injury: Abnormalities in hip an ankle kinematics. Am J Sports Med 37: 252-259, 2009.

6. Brodersen, A, Pedersen, B, and Reimers, J. Incidence of complaints about heel, knee and back related discomfort among Danish children, possible relations to short muscles. Ugeskr Laeger 156: 2243-2245, 1994.

7. Castro-Piñero, J, Chillón, P, Ortega, FB, Montesinos, JL, Sjostrom, M, and Ruiz, R. Criterion-related validity of sit-and-reach and modified sit-and-reach test for estimating hamstring flexibility in children and adolescents aged 6-17 Years. Int J Sports Med 30: 658662, 2009.

8. Coenen, P, Kingma, I, Boot, CRL, et al. Estimation of low back moments from video analysis: A validation study. $J$ Biomech 44 2369-2375, 2011.

9. Coenen, P, Kingma, I, Boot, CR, Bongers, PM, and Dieen, JH. Interrater reliability of a video-analysis method measuring low-back load in a field situation. Appl Ergon 44: 828-834, 2013.

10. Ditmyer, MM, Topp, R, and Pifer, M. Prehabititation in preparation for orthopedic surgery. Orthop Nurs 21: 43-51, 2002.

11. Esola, M, McClure, P, Fitzgerald, G, and Siegler, S. Analysis of lumbar spine and hip motion during forward bending in subjects with and without a history of low back pain. Spine 21: 71-78, 1996.

12. Gajdosik, RL. Effects of static stretching on the maximal length and resistance to passive stretch of short hamstring muscles. $J$ Orthop Sports Phys Ther 14: 250-255, 1991.

13. Gajdosik, R and Lusin, G. Hamstring muscle tightness: Reliability of an active-knee-extension test. Phys Ther 63: 1085-1088, 1983.

14. Hafner, T and Shiffman, J. The emergence of global attention to health systems strengthening. Health Policy Plan 1-10, 2012.

15. Hayes, K, Walton, J, and Szomor, Z. Reliability of five methods for assessing shoulder range of motion. Aust J Physiother 47: 289-294, 2001.

16. Hewett, TE, Torg, JS, and Boden, BP. Video analysis of trunk and knee motion during non-contact anterior cruciate ligament injury in female athletes: Lateral trunk and knee abduction motion are combined components of the injury mechanism. Br J Sports Med 43: 417-422, 2009.

17. Jackson, AW and Baker, AA. The relationship of the sit and reach test to criterion measures of hamstring and back flexibility in young females. Res Q Exerc Sport 58: 183-186, 1986.
18. Kovacs, $M$. The argument against static stretching before sport and physical activity. Athl Ther Today 2: 6-8, 2006.

19. Kraemer, R and Knobloch, K. A soccer-specific balance training program for hamstring muscle and patellar and achiles tendon injuries. Am J Sports Med 37: 1384-1393, 2009.

20. LongoMatch. LongoMatch: The Digital Coach. Fluendo: Barcelona, 2013.

21. Lopez-Miñarro, P, Sainz de Baranda Andujar, P, and RodríguezGarcía, P. A comparison of the spinal posture among several sit-andreach test protocols. J Sports Sci Med 22: 456-462, 2007.

22. Lopez-Miñarro, P, Sainz de Baranda Andujar, P, and RodríguezGarcía, P. A comparison of the sit-and-reach test and the backsaver sit-and-reach test in university students. J Sports Sci Med 8: 116-122, 2009.

23. Maheu, MM, Whitten, $\mathrm{P}$, and Allen, A. E-Health, Telehealth and Telemedicine: A Guide to Startup and Success. San Francisco, CA: Jossey-Bass, 2002.

24. Mier, C. Accuracy and feasibility of video analysis for assessing hamstring flexibility and validity of the sit-and-reach test. Res $Q$ Exerc Sport 82: 617-623, 2011.

25. Mier, C and Shapiro, BS. Reliability of a computer software angle tool for measuring spine and pelvic flexibility during the sit-andreach test. J Strength Cond Res 27: 501-506, 2013.

26. Ogueta-Alday, A, Morante, J, Rodríguez-Marroyo, JA, and GarcíaLópez, J. Validation of a new method to measure contact and flight times during treadmill running. J Strength Cond Res 27: 1455-1462, 2013.

27. Orchard, J. Intrinsic and extrinsic risk factors for muscle strains in Australian football. Am J Sports Med 29: 300-303, 2001.

28. Palacín-Marín, F, Esteban-Moreno, B, Olea, N, Herrera-Viedma, E, and Arroyo-Morales, M. Agreement between telerehabilitation and face-to-face clinical outcome assessments for low back pain in primary care. Spine 38: 947-952, 2013.

29. Puentedura, E, Huijbregts, P, Celeste, S, et al. Immediate effects of quantified hamstring stretching: Hold-relax proprioceptive neuromuscular facilitation versus static stretching. Phys Ther Sport 12: 122-126, 2011.

30. Russell, T, Blumke, R, Richardson, B, and Truter, P. Telerehabilitation mediated physiotherapy assessment of ankle disorder. Physiother Res Int 15: 167-175, 2010.

31. Russell, TG, Buttrum, P, Wootton, R, et al. Rehabilitation after total knee replacement via low-bandwidth telemedicine: The patient and therapist experience. J Telemed Telecare 10: 85-87, 2004.

32. Sjolie, A. Low-back pain in adolescents is associated with poor hip mobility and high body mass index. Scand J Med Sci Sports 14: 168175, 2004.

33. Telfer, S, Woodburn, J, and Turner, D. An ultrasound based noninvasive method for the measurement of intrinsic foot kinematics during gait. J Biomech 47: 1225-1228, 2014.

34. Warburton, DE, Jamnik, VK, Bredin, SS, and Gledhill, N. The Physical Activity Readiness Questionnaire for Everyone (PAR-Q+) and electronic Physical Activity Readiness Medical Examination (ePARMED-X+). Health Fitness Canada 4: 3-17, 2011.

35. Youdas, JW and Krause, DH. Validity of hamstring muscle length assessment during the sit-and-reach test using an inclinometer to measure hip joint angle. J Strenght Cond Res 22: 303-309, 2008. 